\title{
HIV-1 clade promoters strongly influence spatial and temporal dynamics of viral replication in vivo
}

\author{
Mireille Centlivre, ${ }^{1}$ Peter Sommer, ${ }^{1,2}$ Marie Michel, ${ }^{1}$ Raphaël Ho Tsong Fang, ${ }^{3}$ Sandrine Gofflo, ${ }^{4}$ \\ Jenny Valladeau, ${ }^{4}$ Nathalie Schmitt, ${ }^{5}$ Françoise Thierry, ${ }^{6}$ Bruno Hurtrel, ${ }^{3}$ \\ Simon Wain-Hobson, ${ }^{1}$ and Monica Sala ${ }^{1}$

\begin{abstract}
'Unité de Rétrovirologie Moléculaire, ${ }^{2}$ Unité de Biologie Cellulaire du Noyau, and ${ }^{3}$ Unité de Recherche et d'Expertise Physiopathologie des Infections Lentivirales, Institut Pasteur, Paris, France. ${ }^{4}$ Université Claude Bernard, Hôpital Edouard Herriot, Lyon, France.

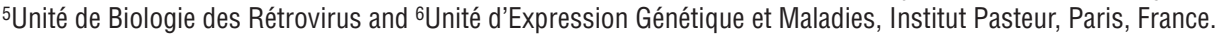

\begin{abstract}
Although the primary determinant of cell tropism is the interaction of viral envelope or capsid proteins with cellular receptors, other viral elements can strongly modulate viral replication. While the HIV-1 promoter is polymorphic for a variety of transcription factor binding sites, the impact of these polymorphisms on viral replication in vivo is not known. To address this issue, we engineered isogenic SIVmac239 chimeras harboring the core promoter/enhancer from HIV-1 clades B, C, and E. Here it is shown that the clade C and E core promoters/enhancers bear a noncanonical activator protein-1 (AP-1) binding site, absent from the corresponding clade $B$ region. Relative ex vivo replication of chimeras was strongly dependent on the tissue culture system used. Notably, in thymic histocultures, replication of the clade $\mathrm{C}$ chimera was favored by IL-7 enrichment, which suggests that the clade $C$ polymorphism in the AP-1 and NF- $\kappa B$ binding sites is involved. Simultaneous infection of rhesus macaques with the 3 chimeras revealed a strong predominance of the clade $\mathrm{C}$ chimera during primary infection. Thereafter, the $\mathrm{B}$ chimera dominated in all tissues. These data show that the clade $\mathrm{C}$ promoter is particularly adapted to sustain viral replication in primary viremia and that cladespecific promoter polymorphisms constitute a major determinant for viral replication.
\end{abstract}

\section{Introduction}

The effect of HIV-1 genetic variation on viral transmission and pathogenesis remains poorly defined. Epidemiological studies have suggested that HIV-1 group M clades might differ in their properties. Epidemiological and phylogenetic studies have also shown that HIV-1 clades are unequally spread out throughout the world, with HIV-1 B being predominant in the West, and HIV-1 C and HIV-1 E in sub-Saharan Africa and Asia (Thailand and India), respectively (http://www.unaids.org). Among the HIV-1 group M viruses, HIV-1 C and HIV-1 E are by far the most prevalent HIVs in the world and are linked to heterosexual transmission. Clade $\mathrm{C}$ has spread rapidly from central Africa down to South Africa and it is now invading regions where other clades preexisted among highrisk populations. Thus, the clade B and $\mathrm{E}$ viruses initially characterized the pandemic in Southeast Asia and India, while B and F were among the first strains in South America. More recently, clade $\mathrm{C}$ has strongly invaded these regions to the point at which it is now dominant in China (http://www.unaids.org).

$\mathrm{HIV}$ and SIV infect various cell types, with $\mathrm{CD} 4^{+} \mathrm{T}$ lymphocytes, macrophages, and DCs being the more frequently infected cell populations in vivo. There is a general consensus that highly pro-

Nonstandard abbreviations used: AP-1, activator protein-1; ds, double-stranded; GALT, gut-associated lymphoid tissue; LC, Langerhans cell; LTR, long terminal repeat; MEF, macrophage-enriched fraction; NFAT, nuclear factor of activated T cells; NRE, negative regulatory element; $3^{\prime} \mathrm{PPT}, 3^{\prime}$ polypurine tract; RhM, rhesus macaque; rPBMC, RhM PBMC; STR, short terminal repeat; TCEF, T cell-enriched fraction; TCID $_{50}, 50 \%$ tissue culture infectious dose; TRE, tetradecanoyl phorbol acetate responsive element. Conflict of interest: The authors have declared that no conflict of interest exists. ductive replication in activated $\mathrm{CD}^{+} \mathrm{T}$ lymphocytes contributes massively to viremia, whereas infected macrophages and DCs constitute reservoirs of clinical relevance $(1,2)$. Yet the influence of local tissue environments on the modulation of viral replication in different cellular subsets in vivo remains largely unknown.

It has been well established that specific HIV/SIV env (the gene encoding the envelope proteins) polymorphisms influence viral infection of particular cell subsets $(3,4)$. Yet viral replication and spread in the host is certainly dependent on events occurring after entry modulation viral latency or productive infection. Accumulating HIV-1 sequence data have shown that the viral promoter is highly polymorphic with clade-specific traits (http://www.hiv.lanl.gov). Indeed, different organization and numbers of transcription factor binding sites characterize each HIV-1 subtype-specific promoter. Hence, recruitment of cellular transcription factors might be at the origin of clade-dependent modulation of viral transcription, directly influencing replication and turnover in cell subsets $(5,6)$.

The HIV-1 promoter is localized in the U3 region of the long terminal repeat (LTR) and can be subdivided into 2 major functional domains: a modulatory region and a downstream core promoter/enhancer (7). The role of the HIV-1 modulatory region (including a negative regulatory element [NRE]) overlapping the $3^{\prime}$ region of nef (the gene encoding the viral negative factor) remains poorly defined $(7,8)$. In contrast, the downstream core promoter/enhancer has been widely studied. The core promoter (spanning the CATA box and 3 Sp 1 transcription factor binding sites) is generally conserved among clades, with the exception of clade $\mathrm{E}$ isolates and some recombinant clade AG strains in which the CATATAA motif is substituted by a CATAAAA sequence (9). One to $3 \mathrm{NF}-\kappa \mathrm{B}$ binding sites characterize different enhancer regions of 
A

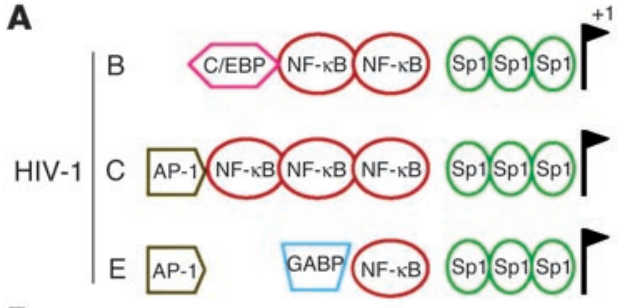

B

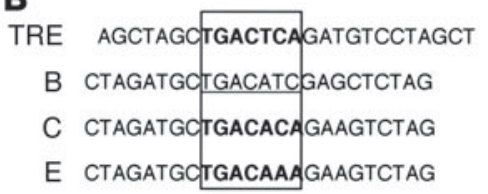

C

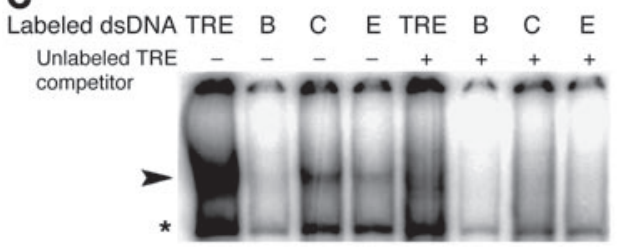

D

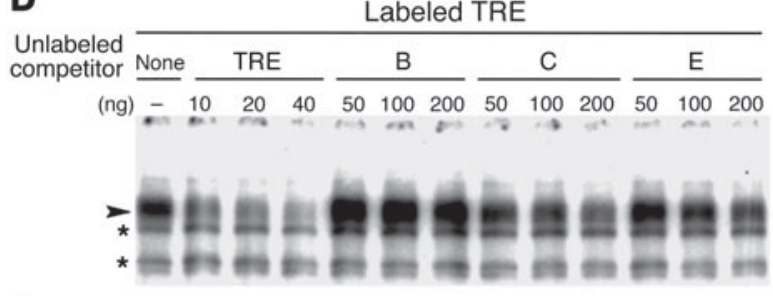

E

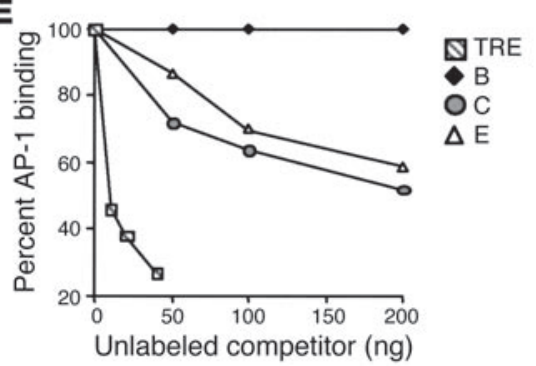

HIV-1 subtypes. The clade C enhancer bears 3 NF- $\mathrm{BB}$ binding sites, while the clade $\mathrm{B}$ isolates are characterized by $2 \mathrm{NF}-\kappa \mathrm{B}$ binding sites and an upstream CCAAT/enhancer binding protein (C/EBP) site (10). In clade E promoters, only $1 \mathrm{NF}-\kappa \mathrm{B}$ binding site is present along with an upstream active GA-binding protein (GABP) site (11) (Figure 1A). In vitro and ex vivo data have shown that the core promoter/enhancer is sufficient to promote viral replication (12) and that host cell type and the activation state of the cell affect viral replication, depending on viral promoter polymorphism (6).

The aim of the present study was to determine in vivo the influence of HIV clade-specific promoter polymorphism on differential viral replication in different tissues. In order to analyze the $\mathrm{B}$, $\mathrm{C}$, and $\mathrm{E}$ core promoters/enhancers in the context of an isogenic in vivo replication-competent virus, we engineered a SIVmac239 clone in which it was possible to freely exchange the viral promoter without altering nef. Based on this genetically modified clone, short terminal repeat (STR) chimeras were generated differing only in their promoter sequences derived from HIV-1 clade B, C, and E prototypes (13). In vitro "mono-infections" with each of the 3 chi-

\section{Figure 1}

Analysis of putative AP-1 binding sites in HIV-1 clade $\mathrm{C}$ and $\mathrm{E}$ promoters/enhancers. (A) Polymorphisms among transcription factor binding sites in the core promoter/enhancer regions of HIV-1 clades B, C, and $E$. The putative clade $C$ and $E$ noncanonical AP-1 binding sites have never before been analyzed experimentally. (B) $5^{\prime}-3^{\prime}$ strands of the TRE, B, C, and E dsDNA used in gel-shift and competition analyses. The TRE of human collagenase 1, bearing a canonical AP-1 binding site (in bold), was used as positive control. $\mathrm{C}$ and $\mathrm{E}$ nucleotide sequences span the putative AP-1 binding sites (in bold) of the HIV-1 clade C and E enhancers, respectively. The clade $B$ sequence spans the homologous sequence (underlined) in the enhancer region of the HIV-1 clade B LTR, used as negative control. TRE is flanked by Alul, and viral sequences, by Xbal (B, C, and E) restriction sites. (C) Gel-shift assays with HeLa nuclear extracts. Arrowhead and asterisk indicate AP-1-specific and nonspecific binding, respectively, to the indicated labeled dsDNA (0.5 $\mathrm{ng}$ ), described in $\mathbf{B}$. The presence of an 80 -fold excess of unlabeled dsDNA TRE competitor (+) is indicated. ( $\mathbf{D}$ and $\mathbf{E}$ ) Gel-shift competition assays with HeLa nuclear extracts. Arrowhead and asterisks indicate AP-1-specific and nonspecific binding, respectively, to the labeled TRE $(0.5 \mathrm{ng})$. Competition with increasing concentrations of the indicated unlabeled dsDNA competitors is shown on a gel (D) and was quantified by ImageQuant and plotted (E).

meras led to replication kinetics comparable to that of the parental SIVmac239 virus. However, ex vivo growth competition kinetics of Chinese rhesus macaque (RhM) PBMCs (rPBMCs) showed that STR variants replicated differently once they were competing in a fixed environment. Moreover, ex vivo coinfection of human thymic histocultures with the 3 chimeras in the presence of IL-7 resulted in massive replication of the clade $\mathrm{C}$ chimera.

The influence of HIV-1 promoter polymorphisms on viral replication was then studied in vivo by coinfection of 2 Chinese RhMs with the B, C, and E STR chimeras. During the 8 months of followup of the infection, blood, serum, and lymph node body compartments were analyzed and, at sacrifice, the relative frequency of STR chimeras was determined in an extensive set of tissues. There was a perfect correlation between data from the 2 coinfected macaques showing a strong predominance of the clade $\mathrm{C}$ chimera during primary infection, followed by the dominance of the clade B chimera in all tissues. The in vivo data, in parallel with in vitro and ex vivo experiments, reveal that the clade $\mathrm{C}$ promoter is particularly adapted to sustain viral replication in primary viremia and that clade-specific promoter polymorphisms constitute a major determinant for viral replication.

\section{Results}

An activator protein-1 binding site is present in the HIV-1 clade C and $E$ enhancers and is absent from clade B. Sequencing of HIV-1 primary isolates (http://www.hiv.lanl.gov) has revealed viral promoter polymorphisms for a variety of transcription factor binding sites with conserved clade-specific features. Notably, in the promoter enhancer just upstream of the NF- $\mathrm{KB}$ binding sites, a noncanonical activator protein-1 (AP-1) binding site has been predicted but not demonstrated in the clade $C$ and $E$ promoters $(6,13)$ (Figure 1A). Thus, we performed gel-shift assays to verify the presence of the AP-1 binding sites using HeLa nuclear extracts $(14,15)$ and double-stranded DNA (dsDNA) oligonucleotides spanning the regions of the HIV-1 C and E enhancers harboring the putative AP-1 binding sites. The homologous region of the HIV-1 B enhancer was used as negative control for AP-1 binding, and the tetradecanoyl phorbol acetate responsive 
element (TRE), bearing a canonical binding site, was used as positive control (Figure 1B). This analysis showed that an AP-1 site was indeed present in clades $\mathrm{C}$ and $\mathrm{E}$ and, as expected, was absent from the clade B promoter (Figure 1, C-E). These data were confirmed using purified JunB/Fra2 and JunB/C-Fos heterodimers (data not shown). In this experimental context, the HIV-1 clade B AP-1 binding site sequence, differing by only 2 nucleotides from the canonical AP- 1 binding site, acted as a mutated AP-1 binding site as it was unable to directly bind AP-1 (Figure 1C) or to compete with the TRE (Figure $1, \mathrm{C}$ and D). Competition experiments allowed determination of the specificity and the strength of AP-1 binding, as in this experimental technique the quantity of nuclear extract and dsDNA competitors are not limiting factors. Even at a 400-fold excess of unlabeled competitor on labeled target for AP-1 direct binding (Figure 1D), it could be shown that AP-1 bound specifically to the clade $\mathrm{C}$ and $\mathrm{E}$ binding sites and that the binding to the clade $\mathrm{C}$ site was at least twice as strong as the binding to the clade E site (Figure 1E).

SIVmac239 chimeras bearing HIV-1 clade-specific promoters are fully replication competent. With the aim of analyzing the effect of promoter polymorphism on viral replication both ex vivo and in vivo, we constructed a SIVmac239 derivative in which it was possible to modify the viral promoter without altering nef(16). In SIVmac239, nef overlaps approximately $80 \%$ of $\mathrm{U} 3$ in the $3^{\prime}$ LTR. This is due to the localization of cis-acting elements that are essential for viral replication within nef: the U-box, the $3^{\prime}$ polypurine tract ( 3 'PPT), and the viral attachment site (att) (17). The extensive overlap limits the possibility of modifying the promoter region without affecting Nef. To overcome this and to prevent possible effects of the SIVmac239 NRE on the core promoter/enhancer, we inactivated the cis-acting elements by introducing 13 silent point mutations in nef and inserted the functional elements between the nef stop codon and the single NF- $\kappa \mathrm{B}$ binding site in the SIVmac239 U3 region (Figure 2). The relocation of the 3 'PPT displaced the $\mathrm{U} 3$ region downstream of nef and resulted in a clone with a short U3 of approximately $110 \mathrm{bp}$, called STR. The SIV core promoter/enhancer was then replaced by the corresponding elements from the U3 of HIV-1 $\mathrm{B}, \mathrm{C}$, and $\mathrm{E}$ (Figure 1A). The promoter sequence from nucleotide -147 to nucleotide -1 of the clade $B$ HIV-1 LAI and the clade C1 and clade E HIV-1 strain analogous sequences (GenBank accession numbers K02013, AF127567, and AF127570, respectively), aligned with the clade B HIV-1 LAI sequence as a reference (13), were cloned through the PspAI and PstI sites in the STR construct (Figure 2). After transient transfection of the CEMx174 cell line (derived from the fusion of a 174 human B cell line and the CEM human $T$ cell line) $(18,19)$, viral stocks of isogenic STR variants referred to as STR-WT, STR-B, STR-C, and STR-E were made, bearing the U3 core promoter/ enhancer of SIVmac239, and clade B, C, and E HIV-1 selected strains, respectively. The replication kinetics of the 4 STR clones were characterized on CEMx174 and rPBMCs pooled from 5 SIVmac239-naive Chinese RhMs (Figure 3, A and B). All STR viruses were replication competent and showed replication kinetics very similar to that of the parental SIVmac239.

Growth competition experiments showed differential fitness. Viral replication kinetics can only discern

Figure 2 gross changes in replication capacity and do not reflect the effect of subtle genetic polymorphisms on the replication rates of viral isolates. Compared with individual replication kinetics in monoinfections, growth competition experiments in cell culture between viral isolates provides a far more sensitive assay $(6,9,20-22)$. In competition experiments, viruses must share vital resources. This inevitably enhances subtle phenotypic differences undetectable in mono-infections. Hence, using pooled rPBMCs, we performed growth competition experiments in which STR variants competed 2 by 2 (B/C, B/E, and C/E) and 3 by 3 (B/C/E) (Figure 4, A-D). For all combinations, from the very first data point (day 3 ) to the last (day 39), the STR-B virus predominated, with relative growth as follows (in descending order): STR-B, STR-C, and STR-E. Even when the relative input ratio of the STR-C variant to the STR-B variant was modified from 1:1 to $19: 1$, the STR-B virus outgrew the competitor, although STR-C was able to catch up with STR-B at intermediate time points (Supplemental Figure 1 and data not shown; supplemental material available online with this article; doi:10.1172/JCI200522873DS1). This unexpected observation was not due to an intrinsic bias in the method used, as we were able to reproducibly detect all 3 variants at equivalent frequencies in 1:1:1 input mixtures, well in correlation with the $50 \%$ tissue culture infectious dose $\left(\mathrm{TCID}_{50}\right)$ values of the stocks. Moreover, control experiments on the 1:19 input mix gave up to $100 \%$ STR-C. Thus, although poorly represented in the input mix, the STR-B was able to outgrow STR-C within the first 3 days of competition.

As a prerequisite to in vivo competition experiments in RhMs, we sought to exclude founder effects on viral replication that could

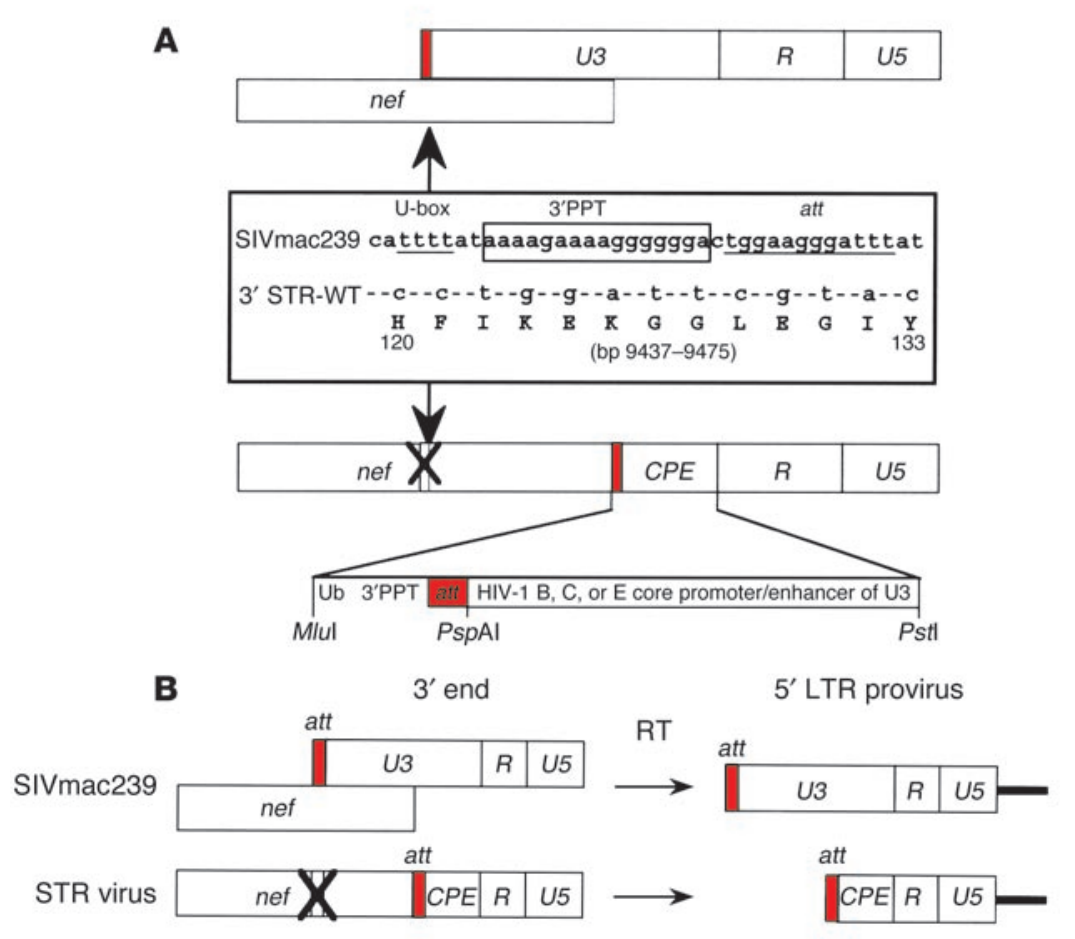

Schematic representation of the STR construct. (A) Silent point mutations in nef, cloning sites, and redesigned region of U3 in the 3'LTR are detailed. Dashes in the 3'STR-WT sequence correspond to the same nucleotides as those of the parental SIVmac239 sequence. Both sequences are localized in their respective genomes at positions indicated by arrows. (B) 3' and 5' LTR structures in SIVmac239 versus homologous regions in the STR chimeras. CPE, core promoter/enhancer. 

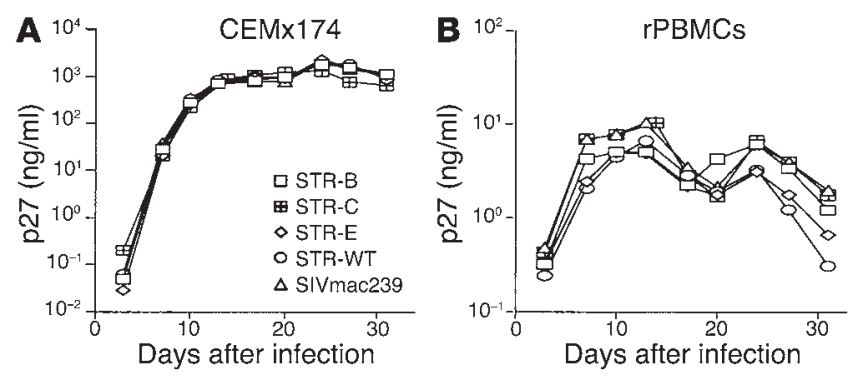

Figure 3

Mean values of replication kinetics assays performed in triplicate. Kinetics assays performed on CEMx174 cells (A) and on rPBMCs obtained from 5 SIV-naive Chinese RhMs, pooled just before infection (B).

be linked to a different RhM genetic background. Accordingly, replication kinetics and growth competition experiments were performed on unpooled rPBMCs from the 2 monkeys chosen for subsequent inoculation. Again, the STR-B virus was predominant in the cultures at all time points tested (Supplemental Figure 2). Hence, the STR chimeras, despite their comparable replication kinetics in mono-infection experiments, revealed strikingly different fitness in growth competition.

IL-7-dependent differential replication of STR chimeras. Growth competition kinetics in rPBMCs showed that STR variants replicate differently once they are competing in a fixed environment. It has been demonstrated that IL-7, along with the TNF- $\alpha$ secreted in the thymic microenvironment, is a critical cytokine for HIV replication in thymocytes, which is regulated at the transcriptional level through NF-кB-dependent activation $(23,24)$. Moreover, in activated human $\mathrm{T}$ lymphocytes, the IL-7 signaling pathway has been shown to be involved in the activation of the Fos and Jun proteins that compose AP-1 (25). Because an AP-1 binding site is present in the STR-C and STR-E promoters and is absent from the STR-B promoter, and the NF-кB binding sites differ in number among the STR chimeras (Figure 1), we set up a competition assay to verify the possible influence of IL-7 on relative replication of STR clade-specific viruses. Hence, this ex vivo growth competition experiment was performed using human thymic histocultures (26) in the presence or absence of IL-7. Cytokine treatment sustained viral replication in thymocytes, as increased p27 viral gag protein levels were only detectable in the presence of IL-7 for up to 21 days of culture (Figure 5A). Analysis of the relative frequency of STR viruses in the culture supernatant showed that STR-C dominated within a few days over STR-B, with STR-E being undetectable from the onset (Figure 5B). Interestingly, the profile of cell-associated viruses did not correspond to that of free virions in the supernatant (Figure 5C). This discrepancy could be explained by apoptosis or necrosis involving the cells sustaining STR-C replication (Figure $5 \mathrm{~B})$, which might have been counterselected from those harboring the less robust STR-B variant (Figure 5C). In conclusion, these data show differential dynamics of replication of the 3 STR chimeras in the presence of IL-7.

Serum- and cell-associated virus differs in primary infection. Although ex vivo studies are useful for studying viral replication in an established environment, they cannot mimic the natural setting within the infected host. Hence, with the aim of analyzing in vivo the influence on viral replication and colonization of the host, we performed coinfection with the STR chimeras of 2 Chinese RhMs. RhMs 97 R0092 and 98R0012 were infected intravenously with 100 TCID $_{50}$ of each of the 3 STR chimeras. Determination of $\mathrm{CD}^{+}$and $\mathrm{CD}^{+} \mathrm{T}$ lymphocyte counts (Figure 6A), plasma RNA viral loads (Figure 6B), and antiSIVmac239 titers (Figure 6C) showed that STR chimeras efficiently infected the 2 macaques. Moreover, infection parameters showed that monkey 97R0092 appeared to be typical for infection in RhMs, with equivalent TCID $_{50}$ of parental SIVmac239, while monkey $98 \mathrm{R} 0012$ infection was markedly atypical because viral RNA levels quickly approach background levels (Figure 6B) (27).

Coinfection with the STR chimeras enabled us to monitor replication in different body compartments using the 3 different promoters as markers. Independently of the capacity of each monkey to control viral RNA levels, this in vivo experiment revealed a perfect correlation between data from the 2 coinfected macaques. The relative proportion of viral DNA present in rPBMCs obtained from both infected monkeys (Figure 7, A and B) was not reflected by that of the circulating viruses from serum (Figure 7, C and D). During the first 2 months of infection, STR-C virus was the predominant

\section{Figure 4}

Growth competition experiments using pooled rPBMCs (each data point is in triplicate). Ex vivo coinfection with STR-B (black bars) and STR-C (gray bars) (A), STR-B and STR-E (white bars) (B), STR-C and STR-E (C), and STR-B, STR-C, and STR-E (D).
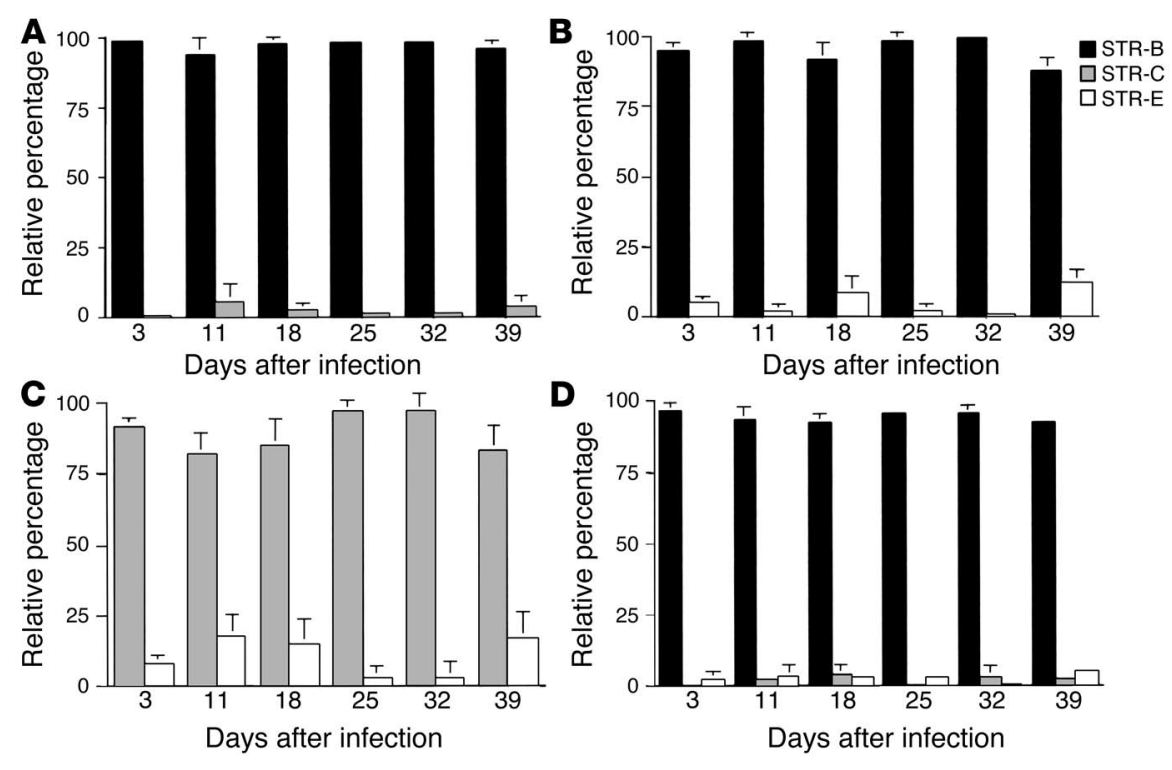

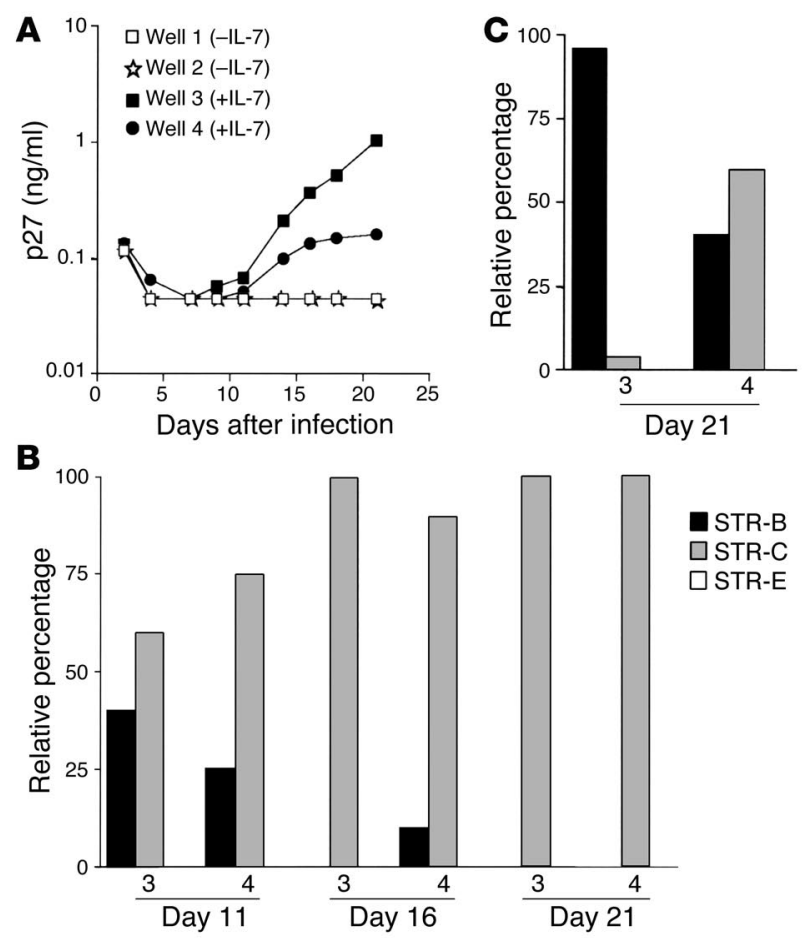

form in the serum, along with the STR-E virus, for both animals. Then a switch to the STR-B form occurred in monkey 97R0092, persisting up to sacrifice (week 36). This distribution was in sharp contrast to the situation in rPBMCs, for which the STR-B virus dominated the early phase of infection in both monkeys. Notwithstanding those results, at peak viremia (day 13), the chimera DNA profile in rPBMCs showed the rebound of the STR-C upon the STR-B form. Then STR-B became again the dominant genotype, with exceptions at weeks 18 and 36, for monkey 97R0092.

At weeks 10, 22, and 31, we investigated the distribution of the 3 STR viruses in peripheral lymph nodes (left and right inguinal and left axillary). At all time points, in macrophage-enriched fractions (MEFs) or CD4 ${ }^{+} \mathrm{T}$ cell-enriched fractions (TCEFs) obtained from lymph nodes, the predominant virus was STR-B, and the relative proportion among variants in TCEFs recalled that of the corresponding rPBMC samples (Figure 7, E and F). In order to identify

\section{Figure 5}

Growth competition experiments using fresh human thymus fragments. (A) Global viral kinetics were performed in duplicate in the absence (wells 1 and 2) or presence (wells 3 and 4) of $5 \mathrm{ng} / \mathrm{ml} \mathrm{IL-7.} \mathrm{(B} \mathrm{and} \mathrm{C)}$ Relative percentage of STR chimeras determined for thymic histoculture supernatants (B) and cells (C) from wells 3 and 4.

recombination or substitution events that might have occurred during infection, the STR regions were analyzed by sequencing the chimeric STR promoters. The 2 sets of primers used exclusively amplify the complete 5' STR promoter regions (see Methods). Analysis of lymph nodes, rPBMCs, and serum at weeks 10, 22, and 31 did not show any mutations (data not shown), confirming that the SIV-STR configuration is genetically stable in vivo, as shown previously for an analogous strain (17).

Persistent STR-C and STR-E despite predominance of STR-B. During the 8 months of infection, access to body compartments was obviously limited. At sacrifice, it was possible to investigate the distribution of the different STR chimeras in an extensive set of tissues (Table 1). In general, cellular fractions were enriched for macrophages and $\mathrm{CD} 4^{+}$ T lymphocytes (MEFs and TCEFs, respectively). It was not possible to recover viral DNA from the jejunum of either animal, although it was possible to amplify CD3 $\gamma$ DNA from the same samples. Interestingly, this part of the gut undergoes the greatest depletion of activated memory $\mathrm{CD}^{+} \mathrm{T}$ lymphocytes (28). As a consequence of active viral replication, infected cells might have been eliminated to undetectable levels. Although the STR-B variant predominated in almost all samples, STR-C and to a lesser extent STR-E were present in many organs analyzed, at relative percentages ranging from 1-29\% (Table 1). Remarkably, the relative proportion between STR-B and STR-C chimeras in rPBMCs did not reflect that of any organ analyzed at sacrifice of monkey 97R0092.

$S T R-B$ predominated in recently infected epithelial Langerhans cells at sacrifice. Langerhans cells (LCs) are of major interest as they might play a primary role in sexual transmission of HIV/SIV infection (29). However, a few publications $(30,31)$ have shown that LC homeostasis is unchanged in skin and vaginal epithelia during HIV/SIV pathogenesis. By HLA-DR staining of skin epidermis from the 2 monkeys and comparison of the data with that in the literature (31), it was possible to show that homeostasis of cutaneous DC populations (LCs and dermal DCs) was not altered during infection
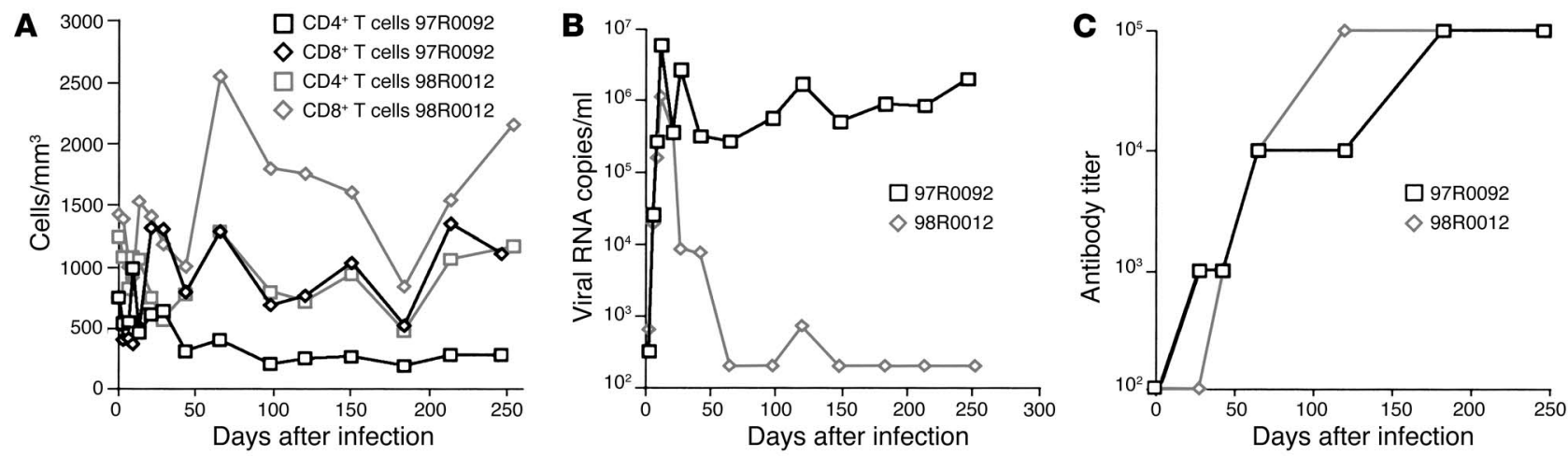

Figure 6

Immunological and virological parameters after infection of RhMs 97R0092 and 98R0012. (A) Blood CD4+ and CD8 ${ }^{+}$T lymphocyte counts. (B) Serum viral RNA loads (lower limit of detection, 200 copies $/ \mathrm{ml}$ ). (C) Serum antibody responses to infection by STR chimeras (lower limit of detection, 100-fold dilution). In C, $y$ axis values represent the reciprocal of experimental values. 
A

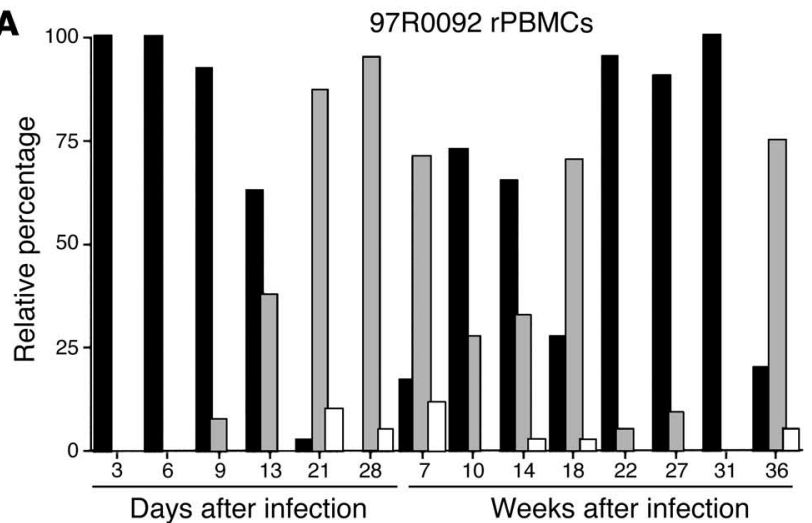

C

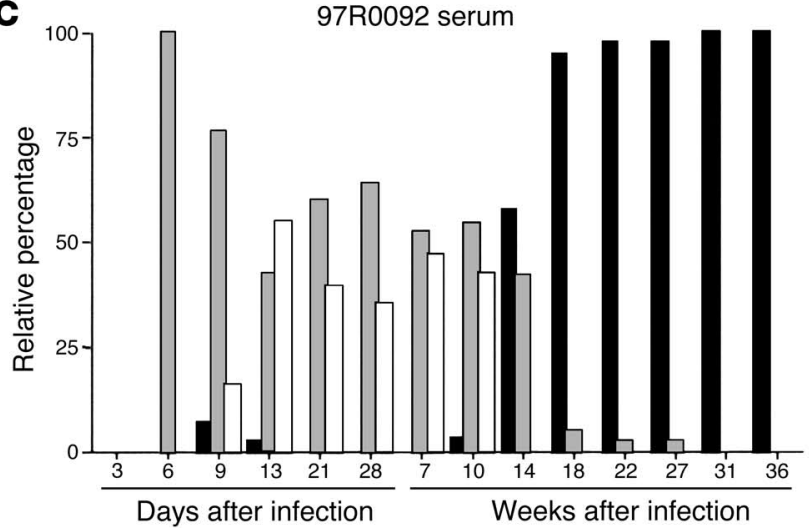

E

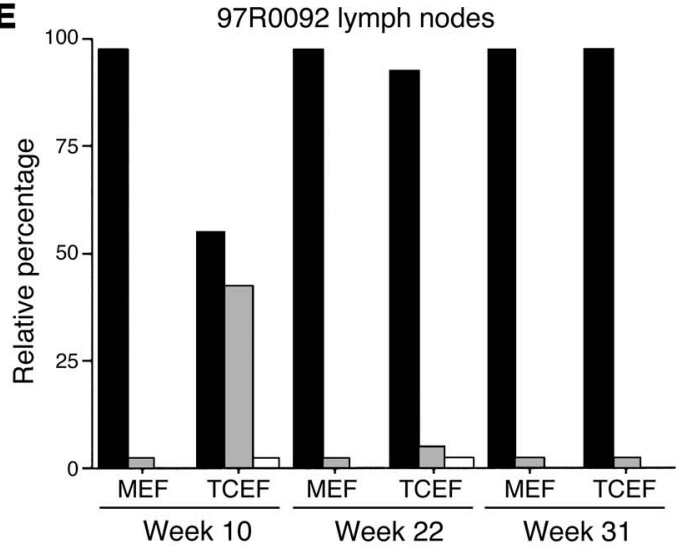

B

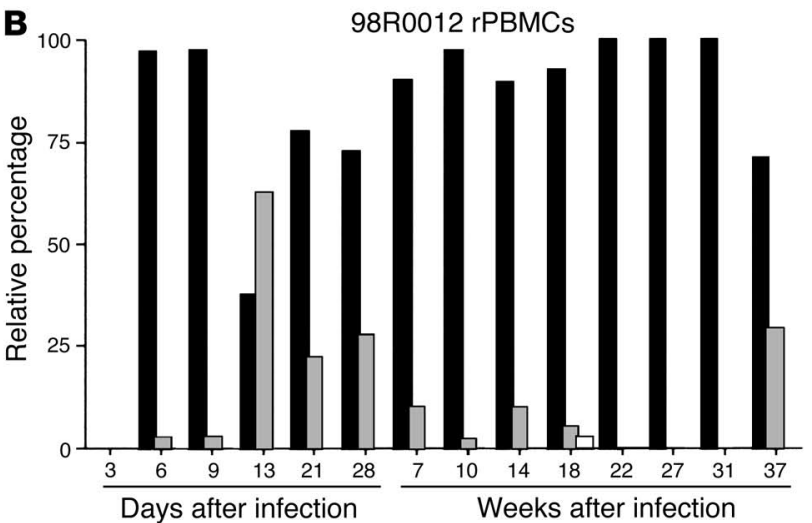

D
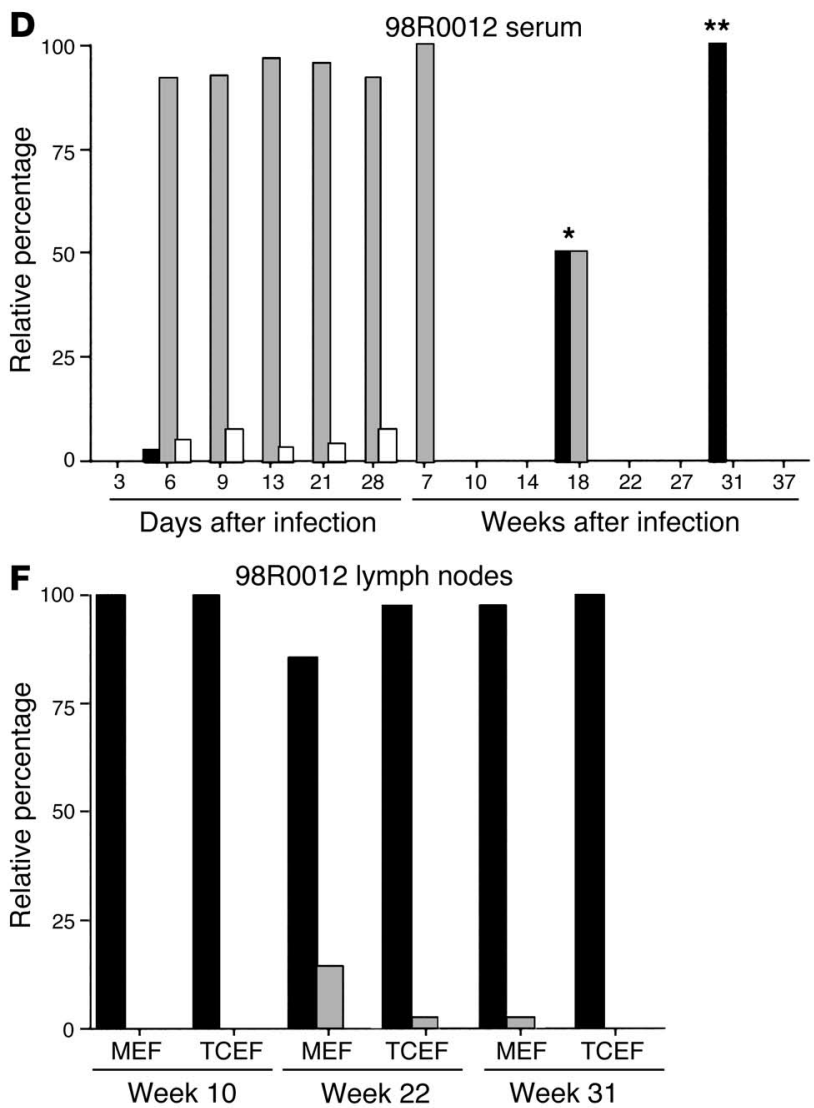

Figure 7

Relative frequency of STR chimeras. Relative frequencies were determined in rPBMCs of monkey $97 \mathrm{R} 0092$ (A) and monkey $98 \mathrm{R} 0012$ (B); in serum of monkey 97R0092 (C) and monkey 98R0012 (D) ( ${ }^{*}$ and ${ }^{* *}$ correspond to a viremia of 740 copies $/ \mathrm{ml}$ and to a value just at the cut-off, respectively); and in MEFs and TCEFs obtained from the lymph nodes of monkey $97 R 0092$ (E) and monkey $98 R 0012$ (F).

(Figure 8, A and D). Indeed, a normal distribution of LCs in skin and vaginal epithelium in both infected animals was shown using $\mathrm{CD} 1 \mathrm{a}$ as a specific marker for LCs (Figure 8, B and E). The skin epidermis was completely devoid of T cells (Figure 8C). Nevertheless, in the vaginas from both RhMs, many $\mathrm{CD} 4^{+} \mathrm{T}$ lymphocytes were found in the lamina propria and few $\mathrm{T}$ cells were found in the epidermis and dermis (Figure 8F). By immunomagnetic bead sorting and propidium iodide exclusion of dead cells, $90 \% \mathrm{LC}$-enriched fractions were obtained from epidermis and vaginal epithelium taken at sacrifice of the infected RhMs (Figure 8, G and H), completely devoid of $\mathrm{CD}^{+}$cells (data not shown). The LC fractions obtained were used to determine the relative proportion of STR viruses in this cell compartment. STR-B predominated in LC fractions derived from skin and vagina of both animals (Table 1). To further characterize LC viral DNA, we sequenced the env V1-V2 regions in parallel with corresponding sequences form rPBMCs at days 21, 120, and 246 (Supplemental Figure 3). For monkey 98R0012, there were so few mutations that useful information could not be obtained (data not shown). However, phylogenetic analysis of sequences from monkey 97R0092 showed that the V1-V2 sequences from vaginal LCs clustered with those from rPBMCs of day 246, suggesting relatively recent infection of those epithelial LCs. We have previously demon- 
strated that human epidermal LCs may be locally infected by underlying infected $T$ cells while their precursors are colonizing the epidermis (32). The present data confirm the notion that LCs analyzed 8 months after infection may not have derived from $\mathrm{CD} 34^{+}$progenitors infected in bone marrow but may have derived from peripheral precursors that are infected while they colonize the epidermis.

\section{Discussion}

Our data here show that HIV-1 M clade-specific promoter polymorphisms translate into strikingly different phenotypes depending upon the environment of viral replication. As the chimeras used in our study are isogenic aside from the promoter, they share the same probability of entering a target cell. After provirus formation, promoter polymorphisms may result in differential replication depending on the cellular environment (6, 13). Our work here provides 3 such examples. First, when propagated individually on CEMx174 or rPBMCs, the chimeras proved to be indistinguishable (Figure 3), whereas when propagated together ex vivo using the same cellular substrates, the STR-B virus dominated, indicative of subtle fitness differences (Figure 4). Second, when growth competition was performed on human thymic histocultures, the STR-C virus dominated in the culture supernatant (Figure 5). Finally, when the competition was performed in vivo, striking temporal fluctuations were observed: STR-C virus dominated in primary infection in both animals and was supplanted by STR-B after seroconversion (Figure 7). Despite this, STR-C persisted and replicated throughout the 8 months of observation, as revealed by V1-V2 sequence analysis (Supplemental Figure 3). Notably, the mutation fixation rate of the V1-V2 env sequences recovered at sacrifice in 97R0092 rPBMCs varied between $1 \times 10^{-2}$ and $1.8 \times 10^{-2}$ substitutions/base/year. Based on estimations made for HIV-1 mean mutation rates in the range of $3.5 \times 10^{-5}$ per base per cycle (33), it can be assumed that STR proviral genomes analyzed at day 246 resulted from 200-340 replication cycles. Hence, the viruses underwent $0.8-1.4$ rounds of replication per day. These values correlate with those determined for kinetics of HIV/SIV replication (34). Wild-type V1-V2 env sequences were $40 \%$ at day 21 and $21 \%$ at week 18 and were absent at sacrifice. As at these time points STR-C massively predominates over STR-B and STR-E (Figure 7A); these data taken together seem to indicate that STR-C proviruses in rPBMCs are the integrated forms of viruses that have been constantly replicating and spreading in specific host niches.

The striking predominance of STR-C in serum, and to a lesser extent, STR-E, during primary infection and their eclipse after seroconversion merit explanation (Figure 7). It is more surprising in that the analysis of the cell-associated virus in rPBMCs and lymph node samples revealed a much higher proportion of STR-B. Sequencing of the 5' STR regions at weeks 10, 22, and 31 from lymph nodes, rPBMCs, and serum showed no significant modification in the promoter regions compared with input STR viruses, excluding the emergence of a recombinant with a replication advantage due to a reorganized promoter, as shown previously (35). This raises the question of where serum or plasma viremia is coming from in primary infection. The differential sensitivity of STR-C to IL-7 in the thymic histocultures (Figure 5) suggests that the polymorphism in the AP- 1 and NF- $\mathrm{KB}$ binding sites may be involved (Figure 1). It has been shown that HIV-1 replication is regulated at the transcriptional level by NF- $\mathrm{BB}$ activity through the thymic microenvironment that provides TNF- $\alpha$,

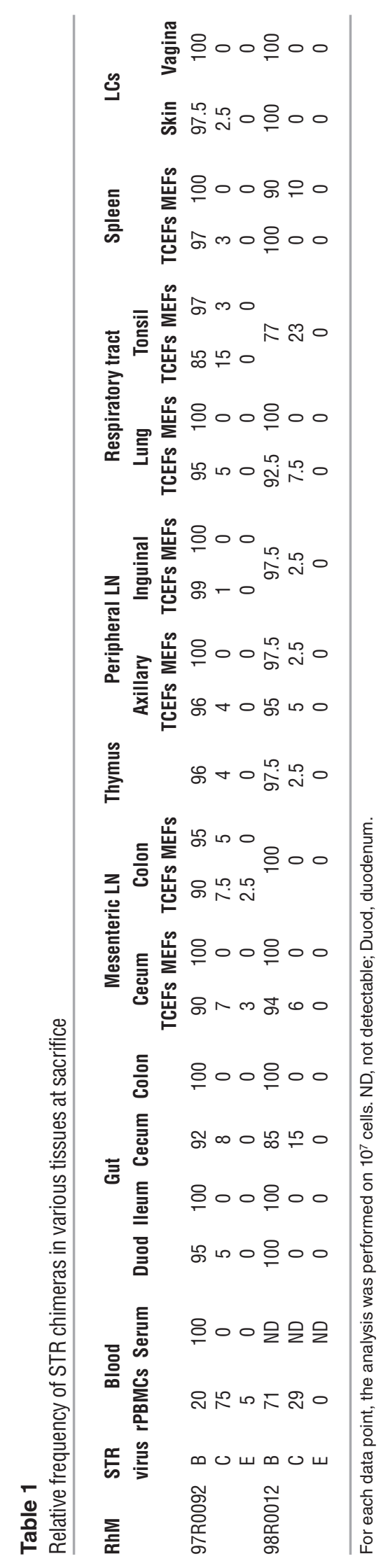



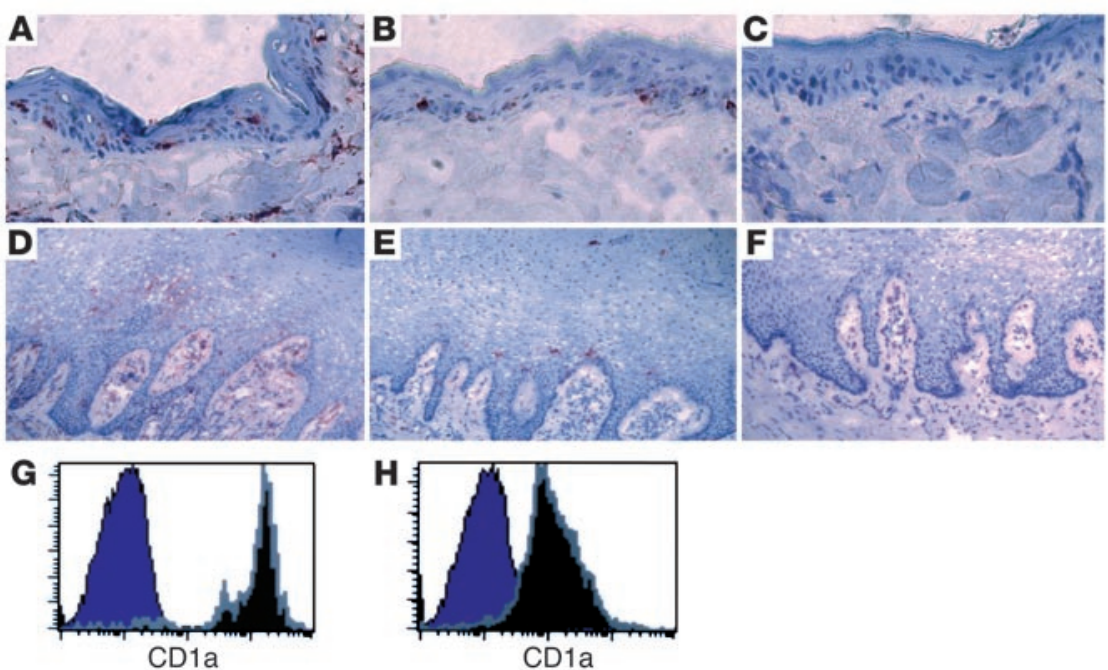

\section{Figure 8}

Immunohistochemical analysis of LCs in skin and vagina. Representative data from monkey 98R0012 are given. (A-F) HLA-DR expression in skin (A) and vagina (D); CD1a expression in skin (B) and vagina (E); and CD3 expression in skin (C) and vagina (F). Magnification, $\times 400$ $(\mathbf{A}-\mathbf{C})$ and $\times 200$ (D-F). (G and H) Representative histograms of $\mathrm{CD} 1 \mathrm{a}^{+}$cells (gray peaks) and CD1a- cells (purple peaks) from LC-enriched fractions in skin $(\mathbf{G})$ and vagina $(\mathbf{H})$.
IL-1, and IL-7 $(23,24)$. Previous studies have also shown that the AP-1 and NF- $\mathrm{\kappa B}$ pathways are activated and that AP- 1 binding is increased in T lymphocytes stimulated with IL-7 (25). Moreover, it has been shown that nuclear factor of activated T cells (NFAT), which binds to NF-KB binding sites, acts synergistically with AP-1 if binding sites are juxtaposed (36). In an environment enriched in IL-7, this could be at the origin of the predominance of STR-C, which harbors a functional AP-1 binding site close to the NF- $\mathrm{BB}$ elements. In contrast, the AP-1 binding site in the STR-E promoter is separated by a spacer of approximately $12 \mathrm{nt}$ from the other enhancer elements, which are a GABP site and a single NF- $\kappa \mathrm{B}$ binding site targeted by Ets and NF-кB/NFAT transcription factors, respectively (37) (Figure 1A). Finally, STR-B completely lacks an AP-1 binding site. Altogether it is tempting to speculate that, in addition to NF- $\mathrm{KB}$ and NFAT, a third key transcription factor differentially targeted by $\mathrm{T}$ cell receptor signaling, namely AP-1, is also able to modulate HIV-1 transcription, with important implications for the spatial and temporal dynamics of viral replication in vivo. Given that the HIV life cycle is tightly linked to the T cell signaling machinery, this implies a means by which this retrovirus can exploit various cellular microenvironments and/or differentiation/activation states for its propagation and maintenance. Moreover, HIV-1 strains have been isolated that completely lack NF- $\mathrm{KB}$ binding sites, and a SIVmac239 mutant containing deletions of all NF- $\mathrm{KB}$ and Sp1 binding sites was shown to replicate with kinetics similar to those of the parental strain in lymphoid cells and caused AIDS in RhMs (38). Hence, the high degree of flexibility in responding to various cellular conditions correlates well with the longstanding notion that the HIV-1 LTR tolerates many mutations in important transcription factor binding sites and possesses high functional redundancy (39). In our study here, the variables were already reduced by genetic separation of the core promoter/enhancer from nef/U3 overlapping sequences. This eliminated about $80 \%$ of the wild-type U3 region containing recognition motifs for myriad DNA-binding factors. Despite this simplification of the promoter, competition experiments were absolutely required to reveal the subtle fitness advantages of a given promoter in a specific environment. Here, in vitro and in vivo data provide the first evidence to our knowledge that AP-1 binding sites present in the promoter can powerfully influence viral replication and fitness. Yet the possibility that the observed growth differences are also influenced by altered cooperativity for NF-кB and/or NFAT binding cannot be excluded. Similarly, it has been shown recently that the subtype E CATAAAA motif confers a slight disadvantage in competition experiments compared with the CATATAA motif present in all other subtypes (9). This might have accounted for the limited activity of the STR-E promoter. Thus, it will be important to further investigate the influence of the main transcription factor response elements in appropriate viral culture systems that allow separation of the pathways leading to activation of AP- $1, \mathrm{NF}-\kappa \mathrm{B}$, and NFAT, an experimentally challenging goal in light of the extensive cross-talk between these key players during $\mathrm{T}$ cell activation.

The transitory nature of STR-C and STR-E viremia suggests that a cellular subset is involved that is rapidly depleted, allowing outgrowth of a more adapted variant (STR-B) in an environment in which IL-7 plays a limited role. The dominance of STR-B thereafter suggests that the compartment that supported STR-C and STR-E growth was not able to reconstitute itself to any significant degree. Given that the extensive analysis of lymphoid tissues was performed at sacrifice, some 8 months after primary viremia, it is not surprising that the sites of extensive STR-C and STR-E replication were not identified. Elucidation of this issue will need analysis during the peak of primary viremia. Nevertheless, it has been shown that like IL-7 (25), IL-15 has the ability to induce AP-1 and NF-אB activation in PBMCs (40). It has also been shown that IL-15, together with the other $\gamma \mathrm{c}$-dependent cytokines, IL-2 and IL-7, regulates the development of $\mathrm{T}$ lymphocyte subsets in the gastrointestinal tract (41). Investigation of SIV infection of RhMs has demonstrated that high viral replication and severe $\mathrm{CD} 4^{+} \mathrm{T}$ lymphocyte depletion occur within the gut-associated lymphoid tissue (GALT). CD4 ${ }^{+} \mathrm{T}$ lymphocytes are almost entirely and irreversibly depleted within 3 weeks of infection. Indeed, depletion of GALT $\mathrm{CD}^{+} \mathrm{T}$ lymphocytes occurs sooner and is by far more extensive than what is seen in peripheral blood, lymph node, or spleen (27, $28)$. Given the results of our study here, as well as published data $(25,27,28,40,41)$, it could well be that the GALT environment results in differential strain replication during primary infection.

Even though the explanation for the predominance of serumborne STR-C in primary viremia is not readily forthcoming, the finding is challenging. Among the HIV-1 M clade viruses, clade $\mathrm{C}$ has spread rapidly from central Africa down to South Africa. 
Furthermore, it is invading regions where other clades preexisted among high-risk populations (http://www.unaids.org). That the promoter of STR-C is apparently better adapted to primary infection could correlate with the global dynamics of clade $\mathrm{C}$ viruses if the pandemic were being driven mainly by individuals with primary infection with high viremia (42). Nevertheless, while our data clearly show that functional promoter differences can affect replication in vivo, they do not exclude the possibility that other viral elements coevolving within each clade may attenuate such differences, such that on average each clade is differently but optimally adapted. Yet it has been extensively demonstrated that the genetic profiles of elements playing a role in viral entry $(22,43)$ and transactivation (44) can differentially modulate the dynamics of the viral life cycle. A particular element, however, may dominate in a specific environment and/or during a particular step of the viral life cycle. Hence, it is possible that the promoter activity plays a major role in differential colonization of tissue compartments, notably in primary infection. Although our data suggest that functional promoter differences mapped ex vivo can affect replication in vivo, this does not a priori indicate that it is the sole determinant. In particular, a critical role for coreceptor usage in pathology has been reported in studies using simian HIVs bearing CCR5 (R5) or CXCR4 (X4) HIV-1 envelopes, which replicated with similar kinetics and at comparable levels as those in monkeys (45). It is noteworthy, however, that both env sequences used in this study (45) derived from clade $B$ viruses. Importantly, in studies using simian HIVs bearing R5tropic env genes from clade B and C viruses, it has been reported that R5-mediated lymphopenia in the gut $\mathrm{CD}^{+}$compartment was likely to be completely independent of HIV-1 entry and hence of env genotypes (46). Thus, even an element as fundamental to the viral life cycle as env can become secondary, leaving the primary role to another viral element affecting replication dynamics.

In our system, important spatial and temporal differences were clearly due to promoter polymorphisms, implying differential interactions among viral promoters and cellular transcription factors in a specific tissue environment in vivo. Viremia, a simple and widely used marker, may arise from viral cycling between different tissues and perhaps subtly different cell subsets, as well as reflecting bursts resulting from antigenic stimulation (47-49). Hence, in addition to entry and transactivation elements, LTR genetic polymorphisms can contribute substantially to the differential replication and dissemination of HIV/SIV in vivo. Studies are currently in progress to identify the source of STR-C virus production in primary infection and to better understand the subtle synergic effect of transcription factors on viral promoters and environments favoring selective rescue of specific viral strains.

\section{Methods}

Gel-shift and competition assays. TRE, B, C, and E dsDNA was obtained by hybridization of B-sense/B-antisense, C-sense/C-antisense, E-sense/ E-antisense, and TRE-sense/TRE-antisense oligonucleotide pairs (for oligonucleotide sequences, see Supplemental Table 1) and was used as described previously $(14,15)$.

STR virus production. The 3' portion of the STR-WT genome (p3'STR-WT)

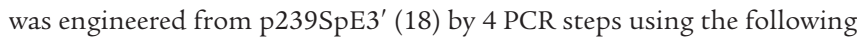
primer pairs: first, 293-3'-8998/293-9680-PPT; second, 239-935-XhoI/239650-MluI; third, STR-MLU/NARSALECOSIV3', and fourth, 293-3'8998/239-650-MluI. By subcloning steps, PspAI and PstI sites were introduced into $\mathrm{p}^{\prime}$ 'STR-WT using primers SPHSMASIV5/NARSALECOSIV3' and PSTSIV5/NARSALECOSIV3'. PCR with primers SMAHIV5/PSTHIV3,
PSPAIHIVC5/PSTHIVC3, and PSPAIHIVE5/PSTHIVE3 on the respective pBlue3'LTR-luc (13) gave the HIV-1 B, C1, and E core promoter/enhancer fragments, which were cloned into p3'STR-WT via PspAI and PstI. Each of the p3'STR plasmids was digested with SphI and ligated to p239Sp5' (18). After transfection with the products of these ligations, STR viruses stocks were prepared on the CEMx174 cell line, derived from the fusion of a 174 human B cell line and the CEM human T cell line (19), and were treated with DNase I (100 U/ml; Amersham). Viral stocks were quantified for antigen p27 with the Coulter SIV Core Antigen Assay kit (Immunotech). $\mathrm{TCID}_{50}$ values were determined as described previously (50).

In vitro and ex vivo replication kinetics and growth competition experiments. Infections were performed in triplicate. CEMx174 $\left(2 \times 10^{6}\right)$ or rPBMCs $\left(3 \times 10^{6}\right)$ that had been pretreated for 2 days with phytohemagglutinin $(2 \mu \mathrm{g} / \mathrm{ml}$; Sigma-Aldrich) and maintained with IL-2 (25 U/ml Gibco; Invitrogen Corp.) were incubated for $3 \mathrm{~h}$ at $37^{\circ} \mathrm{C}$ with $10 \mathrm{ng}$ p 27 of each STR virus. Cells were then extensively washed with PBS and were cultured at a density of $1 \times 10^{6}$ to $1.5 \times 10^{6}$ cells $/ \mathrm{ml}$ in complete RPMI medium (19). In replication kinetics assays performed over 31 days, $0.5 \mathrm{ml}$ of $2 \mathrm{ml}$ of cellfree supernatants was collected and p27 content was quantified. In growth competition experiments, $80 \%$ of the supernatant was withdrawn and half of the cells were collected and resupplied with fresh medium and cells every 2-3 days over a follow-up of 39 days.

Growth competition experiment on thymic histocultures. Thymus was obtained from elective cardiac surgery on an HIV-1-seronegative human newborn. Nine thymus fragments per well were coinfected with a total of 9,000 $\mathrm{TCID}_{50} /$ well $\left(3,000 \mathrm{TCID}_{50}\right.$ per STR virus) of the 3 STR chimeras for 5 hours at $37^{\circ} \mathrm{C}$. Thymus fragments were then maintained as described previously (26). Every 2-3 days, cultures were resupplied with completely fresh medium. Collected supernatants were filtrated and thymus fragments were lysed before determination of the relative frequency of STR viruses.

Infection of RbMs. RhMs (Macaca mulatta) of Chinese origin were serologically negative for SIV, type D retrovirus, herpes B virus, and simian foamy virus. They were housed and cared for in accordance with European guidelines. Blood samples for estimation of $\mathrm{CD}^{+}$and $\mathrm{CD} 8^{+} \mathrm{T}$ lymphocytes counts by flow cytometry analysis were treated as described previously (51) with antibodies against CD3, CD4, CD8, and CD20 (Supplemental Table 2). Viral RNA copies per $\mathrm{ml}$ were measured by real-time quantitative RT-PCR (52). SIV-specific antibody responses were determined as described previously (19).

Relative frequency of STR chimeras. CEMx174 and rPBMC samples from in vitro infections were washed in PBS before lysis as described previously (32). The rPBMCs from in vivo coinfection were purified from blood on a Ficoll gradient (Amersham). One-third of RNA extracted from $250 \mu \mathrm{l}$ of serum was reverse transcribed by avian myeloblastosis virus (AMV RT; Promega) using the primer R3OUT. Of $30 \mu \mathrm{l}$ of this reaction, $10 \mu \mathrm{l}$ was amplified with STRSMAP5/R3OUT for the first PCR round and BCE5ON/R3ON for the second. Organs were homogenized and resuspended in complete RPMI medium. To obtain TCEFs and MEFs, $3 \times 10^{6} \mathrm{cell} / \mathrm{s} / \mathrm{ml}$ were layered on Petri dishes for 1 hour at $37^{\circ} \mathrm{C}$ in complete RPMI medium. Then the nonadherent cell fraction was collected (TCEFs), while MEFs were recovered by scraping of adherent cells (53). After flow cytometry analysis, MEFs showed enrichment in macrophages of at least 2 to 5 times that of TCEFs. Pulmonary and splenic homogenates before adhesion and rPBMCs were purified on a Ficoll gradient (Amersham). All these samples were then subjected to nested PCR spanning the HIV-1 promoter sequences coupled to screening by clade-specific radiolabeled probes. The 5' STR was then amplified with STRSMAP5/ gag1201 for the first PCR round and BCE5ON/NARSIV3 for the second. PCR products were purified on gels, extracted with a Qiagen Extraction kit, and cloned using the TOPO TA cloning kit (Invitrogen Corp.). An average of 40 white colonies were picked from 3 replica plates and were transferred 
to nitrocellulose membrane filters (Schleicher $\&$ Schuell). Filters were selectively hybridized with $\gamma^{-32} \mathrm{P}$-labeled clade-specific probes (COMPB, PSPAIHIVC5, and ECOMP, for STR-B, STR-C, and STR-E, respectively), allowing relative percentage determination of clade-specific STR chimeras.

Sequencing analysis. STR promoter/enhancer regions were amplified by nested PCR with STRSMAP5/gag1201 for the first PCR round and BCE5ON/ NARSIV3 for the second. V1-V2 sequences were obtained by amplification of SIV DNA using the primers SIVOUT5239/C3OUT3 for the first round of PCR and SIVIN5/C3IN3 for the second. Amplified products were purified on gels, extracted with a Qiagen Extraction kit, and cloned using the TOPO TA cloning kit (Invitrogen Corp.). An average of 40 white colonies for the promoter/enhancer and 19-26 for the V1-V2 regions were sequenced and aligned by Clustalw1.83. Sequences of $600 \mathrm{bp}$ spanning the V1-V2 regions were subjected to SplitsTree2.3.1 analysis as described previously (54).

Isolation of LCs. Skin slices from legs and arms were incubated overnight at $4^{\circ} \mathrm{C}$ in $0.1 \%$ trypsin (Sigma-Aldrich). Biopsies of vaginal mucosal tissues were incubated for 90 minutes at $37^{\circ} \mathrm{C}$ in $1 \mathrm{mg} / \mathrm{ml}$ collagenase IV S (SigmaAldrich) and then overnight at $4^{\circ} \mathrm{C}$ in trypsin-EDTA $1 \mathrm{X}$ (Invitrogen Corp.). Epidermis was then separated from dermis with fine forceps. Epidermal fragments were pooled in Hank's balanced salt solution (Invitrogen Corp.) supplemented with $10 \%$ fetal calf serum and were subjected to rounds of pipetting and filtration through sterile gauzes. LCs were enriched on a Ficoll gradient and then were positively purified by 2 rounds of immunomagnetic sorting: 30 minutes of incubation at $4^{\circ} \mathrm{C}$ with anti CD1a and 15 minutes of incubation with bead-conjugated anti-mouse immunoglobulin (MACS reagent kit; Miltenyi Biotec). For flow cytometry analyses, cells were incubated for 30 minutes at $4{ }^{\circ} \mathrm{C}$ with a goat anti-mouse IgG. Indirect immunohistochemistry staining was performed with monoclonal antibodies (Supplemental Table 2) and was visualized using a labeled streptavidinbiotin-peroxidase assay (LSAB2 Kit; Dako).

\section{Acknowledgments}

This paper is dedicated to the memory of Bruno Hurtrel. We would like to thank Susanna Celli, Rémi Cheynier, Colette Dezutter-Dambuyant, Elisabetta Dondi, Nicole Israel, Nicolas Legrand, and Mike McCune for discussions, technical support, and comments on the manuscript. We would also like to thank the NIH for providing pBlue3'LTR-lucB, pBlue3'LTR-lucC, and pBlue3'LTRlucE (13). This research was supported by the Institut Pasteur, Agence Nationale de Recherches sur le SIDA (ANRS), and Centre National de la Recherche Scientifique (CNRS). M. Centlivre and P. Sommer were recipients of ANRS fellowships, and N. Schmitt of a SIDACTION fellowship. M. Centlivre has developed this work in the context of her thesis at the University of Paris VI.

Received for publication July 30, 2004, and accepted in revised form December 12, 2004.

Address correspondence to: Monica Sala, Unité de Rétrovirologie Moléculaire, CNRS UFA1930, Institut Pasteur, 28, rue du Dr Roux, 75015 Paris, France. Phone: 33-140-61-33-09; Fax: 33-14568-88-74; E-mail: joo@pasteur.fr.

Bruno Hurtrel is deceased.
1. McIlroy, D., et al. 1995. Infection frequency of dendritic cells and CD4+ T lymphocytes in spleens of human immunodeficiency virus-positive patients. J. Virol. 69:4737-4745.

2. Collman, R.G., Perno, C.F., Crowe, S.M., Stevenson, M., and Montaner, L.J. 2003. HIV and cells of macrophage/dendritic lineage and other non-T cell reservoirs: new answers yield new questions. J. Leukoc. Biol. 74:631-634.

3. Pierson, T.C., and Doms, R.W. 2003. HIV-1 entry and its inhibition. Curr. Top. Microbiol. Immunol. 281:1-27.

4. Fulcher, J.A., et al. 2004. Compartmentalization of human immunodeficiency virus type 1 between blood monocytes and CD4+ T cells during infection. J. Virol. 78:7883-7893.

5. Roof, P., et al. 2002. Differential regulation of HIV-1 clade-specific B, C, and E long terminal repeats by NF-kappaB and the Tat transactivator. Virology. 296:77-83.

6. Van Opijnen, T., et al. 2004. Human immunodeficiency virus type 1 subtypes have a distinct Long Terminal Repeat that determines the replication rate in a host-cell-specific manner. J. Virol. 78:3675-3683.

7. Roebuck, K.A., and Saifuddin, M. 1999. Regulation of HIV-1 transcription. Gene Expr. 8:67-84.

8. Lu, Y., et al. 1990. Identification of cis-acting repressive sequences within the negative regulatory element of human immunodeficiency virus type 1 . J. Virol. 64:5226-5229.

9. van Opijnen, T., Kamoschinski, J., Jeeninga, R.E., and Berkhout, B. 2004. The human immunodeficiency virus type 1 promoter contains a CATA box instead of a TATA box for optimal transcription and replication. J. Virol. 78:6883-6890.

10. Henderson, A.J., Zou, X., and Calame, K.L. 1995. $\mathrm{C} / \mathrm{EBP}$ proteins activate transcription from the human immunodeficiency virus type 1 long terminal repeat in macrophages/monocytes. J. Virol. 69:5337-5344.

11. Verhoef, K., Sanders, R.W., Fontaine, V., Kitajima, S., and Berkhout, B. 1999. Evolution of the human immunodeficiency virus type 1 long terminal repeat promoter by conversion of an NF-kappaB enhancer element into a GABP binding site.J. Virol. 73:1331-1340.

12. Naghavi, M.H., Schwartz, S., Sonnerborg, A., and Vahlne, A. 1999. Long terminal repeat promoter/ enhancer activity of different subtypes of HIV type 1. AIDS Res. Hum. Retroviruses. 15:1293-1303.

13. Jeeninga, R.E., et al. 2000. Functional differences between the long terminal repeat transcriptional promoters of human immunodeficiency virus type 1 subtypes A through G. J. Virol. 74:3740-3751.

14. Bouallaga, I., Teissier, S., Yaniv, M., and Thierry, F. 2003. HMG-I(Y) and the CBP/p300 coactivator are essential for human papillomavirus type 18 enhanceosome transcriptional activity. Mol. Cell. Biol. 23:2329-2340.

15. Thierry, F., Spyrou, G., Yaniv, M., and Howley, P. 1992. Two AP1 sites binding JunB are essential for human papillomavirus type 18 transcription in keratinocytes. J. Virol. 66:3740-3748.

16. Kestler III, H.W., et al. 1991. Importance of the nef gene for maintenance of high virus loads and for development of AIDS. Cell. 65:651-662.

17. Münch, J., et al. 2001. Simian immunodeficiency virus in which nef and U3 sequences do not overlap replicates efficiently in vitro and in vivo in Rhesus macaques. J. Virol. 75:8137-8146.

18. Kestler, H., et al. 1990. Induction of AIDS in Rhesus monkeys by molecularly cloned simian immunodeficiency virus. Science. 248:1109-1112.

19. Blancou, P., et al. 2004. Simian immunodeficiency virus promoter exchange results in a highly attenuated strain that protects against uncloned challenge virus. J. Virol. 78:1080-1092.

20. Quiñones-Mateu, M., and Arts, E.J. 2001. HIV-1 fitness: implications for drug resistance, disease progression, and global epidemic evolution. In HIV sequence compendium. C. Kuiken et al., editors. Theoretical Biology and Biophysics Group, Los Alamos National Laboratory. Los Alamos, New Mexico, USA. $134-170$.
21. Painter, S.L., Biek, R., Holley, D.C., and Poss, M. 2003. Envelope variants from women recently infected with clade A human immunodeficiency virus type 1 confer distinct phenotypes that are discerned by competition and neutralization experiments. J. Virol. 77:8448-8461.

22. Ball, S.C., et al. 2003. Comparing the ex vivo fitness of CCR5-tropic human immunodeficiency virus type 1 isolates of subtypes B and C. J. Virol. 77:1021-1038.

23. Chene, L., et al. 1999. Thymocyte-thymic epithelial cell interaction leads to high-level replication of human immunodeficiency virus exclusively in mature CD4(+) CD8(-) CD3(+) thymocytes: a critical role for tumor necrosis factor and interleukin-7. J. Virol. 73:7533-7542.

24. Chene, L., Nugeyre, M.T., Barre-Sinoussi, F., and Israel, N. 1999. High-level replication of human immunodeficiency virus in thymocytes requires NF-kappaB activation through interaction with thymic epithelial cells. J. Virol. 73:2064-2073.

25. Gringhuis, S.I., de Leij, L.F., Verschuren, E.W., Borger, P., and Vellenga, E. 1997. Interleukin-7 upregulates the interleukin-2-gene expression in activated human $T$ lymphocytes at the transcriptional level by enhancing the DNA binding activities of both nuclear factor of activated T cells and activator protein-1. Blood. 90:2690-2700.

26. Glushakova, S., Baibakov, B., Margolis, L.B., and Zimmerberg, J. 1995. Infection of human tonsil histocultures: a model for HIV pathogenesis. Nat. Med. 1:1320-1322.

27. Ling, B., et al. 2002. SIV(mac) pathogenesis in rhesus macaques of Chinese and Indian origin compared with primary HIV infections in humans. AIDS. 16:1489-1496.

28. Veazey, R.S., et al. 1998. Gastrointestinal tract as a major site of CD4+ T cell depletion and viral replication in SIV infection. Science. 280:427-431.

29. Miller, C.J., and Shattock, R.J. 2003. Target cells in vaginal HIV transmission. Microbes Infect. 5:59-67.

30. Zimmer, M.I., et al. 2002. Disrupted homeostasis 
of Langerhans cells and interdigitating dendritic cells in monkeys with AIDS. Blood. 99:2859-2868.

31. Ma, Z., Lu, F.X., Torten, M., and Miller, C.J. 2001. The number and distribution of immune cells in the cervicovaginal mucosa remain constant throughout the menstrual cycle of rhesus macaques. Clin. Immunol. 100:240-249.

32. Sala, M., et al. 1994. Spatial discontinuities in human immunodeficiency virus type 1 quasispecies derived from epidermal Langerhans cells of a patient with AIDS and evidence for double infection. J. Virol. 68:5280-5283.

33. Mansky, L.M., and Temin, H.M. 1995. Lower in vivo mutation rate of human immunodeficiency virus type 1 than that predicted from the fidelity of purified reverse transcriptase. J. Virol. 69:5087-5094.

34. Pelletier, E., Saurin, W., Cheynier, R., Letvin, N.L., and Wain-Hobson, S. 1995. The tempo and mode of SIV quasispecies development in vivo calls for massive viral replication and clearance. Virology. 208:644-652.

35. Blackard, J.T., et al. 1999. Transmission of human immunodeficiency type 1 viruses with intersubtype recombinant long terminal repeat sequences. Virology. 254:220-225.

36. Macian, F., Lopez-Rodriguez, C., and Rao, A. 2001. Partners in transcription: NFAT and AP-1. Oncogene. 20:2476-2489.

37. Bassuk, A.G., Anandappa, R.T., and Leiden, J.M. 1997. Physical interactions between Ets and NF-kappaB/NFAT proteins play an important role in their cooperative activation of the human immunodeficiency virus enhancer in T cells. J. Virol. 71:3563-3573.
38. Ilyinskii, P.O., Simon, M.A., Czajak, S.C., Lackner, A.A., and Desrosiers, R.C. 1997. Induction of AIDS by Simian Immunodeficiency Virus lacking NF-кB and Sp1 binding elements. J. Virol. 71:1880-1887.

39. Sommer, P., et al. 2004. Anti-termination by SIV Tat requires flexibility of the nascent TAR structure. J. Mol. Biol. 344:11-28.

40. McDonald, P.P., Russo, M.P., Ferrini, S., and Cassatella, M.A. 1998. Interleukin-15 (IL-15) induces NF-kappaB activation and IL-8 production in human neutrophils. Blood. 92:4828-4835.

41. Porter, B.O., and Malek, T.R. 2000. Thymic and intestinal intraepithelial $\mathrm{T}$ lymphocyte development are each regulated by the gammac-dependent cytokines IL-2, IL-7, and IL-15. Semin. Immunol. 12:465-474.

42. Pilcher, C.D., et al. 2004. Frequent detection of acute primary HIV infection in men in Malawi. AIDS. 18:517-524.

43. Rangel, H.R., et al. 2003. Role of the human immunodeficiency virus type 1 envelope gene in viral fitness. J. Virol. 77:9069-9073.

44. Reza, S.M., et al. 2003. A naturally occurring substitution in human immunodeficiency virus Tat increases expression of the viral genome. J. Virol. 77:8602-8606

45. Harouse, J.M., Gettie, A., Tan, R.C., Blanchard, J., and Cheng-Mayer, C. 1999. Distinct pathogenic sequela in rhesus macaques infected with CCR5 or CXCR4 utilizing SHIVs. Science. 284:816-819.

46. Chen, Z., et al. 2002. CD4+ lymphocytopenia in acute infection of Asian macaques by a vaginally transmissible subtype-C, CCR5-tropic simian/ human immunodeficiency virus (SHIV). J. Acquir.
Immune Defic. Syndr. 30:133-145

47. Ho, D.D. 1992. HIV-1 viraemia and influenza. Lancet. 339:1549.

48. Staprans, S.I., et al. 1995. Activation of virus replication after vaccination of HIV-1-infected individuals. J. Exp. Med. 182:1727-1737.

49. Cheynier, R., et al. 1998. Antigenic stimulation by BCG vaccine as an in vivo driving force for SIV replication and dissemination. Nat. Med. 4:421-427.

50. Johnson, V.A., and Byington, R.E. 1990. Quantitative assays for virus infectivity. In Techniques in HIV research. A. Aldovini, and B.D. Walker, editors. Stockton Press. New York, New York, USA. 71-76.

51. Monceaux, V., Ho Tsong Fang, R., Cumont, M.C., Hurtrel, B., and Estaquier, J. 2003. Distinct cycling CD4(+)- and CD8(+)-T-cell profiles during the asymptomatic phase of simian immunodeficiency virus SIVmac251 infection in rhesus macaques. J. Virol. 77:10047-10059.

52. Hofmann-Lehmann, R., et al. 2000. Sensitive and robust one-tube real-time reverse transcriptasepolymerase chain reaction to quantify SIV RNA load: comparison of one- versus two-enzyme systems. AIDS Res. Hum. Retroviruses. 16:1247-1257.

53. Kuwana, M., et al. 2003. Human circulating CD14+ monocytes as a source of progenitors that exhibit mesenchymal cell differentiation. J. Leukoc. Biol. 74:833-845.

54. Cheynier, R., Kils-Hutten, L., Meyerhans, A., and Wain-Hobson, S. 2001. Insertion/deletion frequencies match those of point mutations in the hypervariable regions of the simian immunodeficiency virus surface envelope gene. J. Gen. Virol. 82:1613-1619. 